\title{
Mesoscale dynamics on the Sun's surface from HINODE observations
}

\author{
Th. Roudier ${ }^{1}$, M. Rieutord ${ }^{1,2}$, D. Brito ${ }^{1,3}$, F. Rincon ${ }^{2}$, J. M. Malherbe ${ }^{4}$, N. Meunier ${ }^{5}$, T. Berger ${ }^{6}$, and Z. Frank ${ }^{6}$ \\ ${ }^{1}$ Laboratoire d'Astrophysique de Toulouse-Tarbes, Université de Toulouse, CNRS, 57 avenue d'Azereix, 65000 Tarbes, France \\ e-mail: roudier@ast.obs-mip.fr \\ 2 Laboratoire d'Astrophysique de Toulouse-Tarbes, Université de Toulouse, CNRS, 14 avenue Edouard Belin, \\ 31400 Toulouse, France \\ 3 Laboratoire de Modélisation et d'Imagerie en Géosciences, Université de Pau et des Pays de l'Adour, CNRS, \\ Avenue de l'Université, 64013 Pau Cedex, France \\ ${ }^{4}$ LESIA, Observatoire de Paris, Section de Meudon, 92195 Meudon, France \\ 5 LAOG, CNRS, Université Joseph Fourier, BP 43, 38041 Grenoble Cedex, France \\ ${ }^{6}$ Lockheed Martin Advance Technology Center, Palo Alto, CA, USA
}

Received 7 October 2008 / Accepted 16 December 2008

\section{ABSTRACT}

\begin{abstract}
Aims. The interactions of velocity scales on the Sun's surface, from granulation to supergranulation are still not understood, nor are their interaction with magnetic fields. We thus aim at giving a better description of dynamics in the mesoscale range which lies between the two scales mentioned above.

Methods. We analyse a $48 \mathrm{~h}$ high-resolution time sequence of the quiet Sun photosphere at the disk center obtained with the Solar Optical Telescope onboard Hinode. The observations, which have a field of view of $100^{\prime \prime} \times 100^{\prime \prime}$, typically contain four supergranules. We monitor in detail the motion and evolution of granules as well as those of the radial magnetic field.

Results. This analysis allows us to better characterize Trees of Fragmenting Granules issued from repeated fragmentation of granules, especially their lifetime statistics. Using floating corks advected by measured velocity fields, we show their crucial role in the advection of the magnetic field and in the build up of the network. Finally, thanks to the long duration of the time series, we estimate that the turbulent diffusion coefficient induced by horizontal motion is approximately $430 \mathrm{~km}^{2} \mathrm{~s}^{-1}$.

Conclusions. These results demonstrate that the long living families contribute to the formation of the magnetic network and suggest that supergranulation could be an emergent length scale building up as small magnetic elements are advected and concentrated by TFG flows. Our estimate for the magnetic diffusion associated with this horizontal motion might provide a useful input for mean-field dynamo models.
\end{abstract}

Key words. Sun: atmosphere - Sun: granulation - Sun: magnetic fields

\section{Introduction}

The transport and evolution of magnetic flux over the solar surface are linked to the turbulent flows developing within the solar convection zone. Indeed, magnetic fields are observed in complex and hierarchical structures covering widely different scales which emerge and disappear on the time-scales of turbulent convective patterns. This continual reconfiguration of surface magnetism by surface flows plays a significant role in determining the topology and evolution of coronal magnetic fields. It may notably influence the triggering of eruptive events such as flares or sudden disappearance of solar filaments. Hence, understanding the distribution and transport of magnetic flux at the solar surface is of primary importance to describe many aspects of solar magnetism.

To reproduce the surface distribution of magnetic flux, different types of processes are included in models: active regions dynamics, differential rotation, meridional circulation and diffusion across the supergranular network (Ossendrijver 2003). Supergranules are known to dominate the evolution and diffusion of surface magnetic fields underlying coronal and heliospheric fields, as evidenced by the structure of both active and quiet magnetic networks. They are distributed over the entire solar surface, indicating that the corresponding velocity field is always present to carry magnetic flux. Moreover, evidence of connections between supergranulation, interplanetary magnetic field and solar energetic particules has been demonstrated with measurements of ions from the impulsive solar Flare with the advanced composition explorer (ACE) spacecraft and numerical simulations of the propagation of energetic particles (Giacalone et al. 2000). Solar wind outflow sources have also been located, with the SUMER/SOHO instrument, along the boundaries and boundary intersections of the magnetic network, which delineates supergranulation (Hassler et al. 1999). More recently, Aiouaz (2008) reported evidence of continuous reconnections at the boundaries of the supergranular network lanes in the quiet Sun and coronal holes. He proposed a scenario involving reconnections between the strong network magnetic field at supergranule boundaries and the continuously advected weak field from the supergranular cell interior. From many points of view, it therefore seems essential to have a good understanding of supergranular flows to construct accurate models of surface and atmospheric magnetic activity of the Sun.

Supergranulation is usually assumed to be the surface imprint of deeper convective motion. Why this scale is so prominent amongst all the continuum of scales remains unexplained. 
As the convection zone is strongly turbulent and highly stratified in density, numerical modelling reaching supergranular scales has proven difficult so far (DeRosa \& Toomre 2004; Rincon et al. 2005; Georgobiani et al. 2007) so that the dynamics at these scales remain poorly understood. Supergranules are traditionally described as convection eddies with horizontal flows diverging from a cell centre and subsiding flows at the cell boundaries and horizontal currents associated with each supergranule are believed to sweep magnetic fields to its borders. Different theoretical approaches try to explain the driving of supergranulation (see a summary in DeRosa \& Toomre 2004). For instance, the spatial correlations between exploding granules may drive a large-scale instability injecting energy at supergranular scales (Rieutord et al. 2000); another scenario suggests that granules impose fixed thermal flux boundary conditions, triggering a convection flow at scales larger than granulation (Rincon \& Rieutord 2003). Rast (2003) suggested that interacting downwards flows cluster and produce the scale of meso or supergranules. The picture is further complicated by the results of Gizon et al. (2003) and Schou (2003) who suggested that supergranulation was associated with a wave pattern; Rast et al. (2004) and Lisle et al. (2004), using a similar approach, however argued that the spectrum of supergranulation was instead consistent with two nonoscillatory flows identified with the mesogranular and supergranular scales having different rotation rates. Overall, it is clear that the physical nature of supergranulation is still in debate and that new accurate measurements are required in order to obtain more clues regarding its physical origin. Addressing this problem most notably requires high-resolution observations of the solar surface dynamics over a large field-of-view and for several consecutive hours.

In this paper, we study photospheric motion using new data obtained with the Hinode satellite, that fulfill some of these requirements. We particularly focus on their relation to the magnetic field network, by identifying and following tree of fragmenting granules (TFGs) introduced by Roudier et al. (2003). These features, also called families of granules, are an appropriate tool to quantify the temporal and spatial organization of the solar granulation at larger scales. In Sect. 2 we describe an exceptionally long (48 h), multi-wavelength, time-sequence of observations obtained with Hinode/SOT. New statistics on TFGs are given in Sect. 3. Section 4 describes a correlation between the photospheric network and the evolution of a passive scalar and magnetic field location. Our conclusions are presented in Sect. 5.

\section{Observations and data reduction}

We used multi-wavelength data sets of the solar optical telescope (SOT), aboard the Hinode ${ }^{1}$. mission (e.g. Suematsu et al. 2008; Ichimoto et al. 2004). The SOT has a $50 \mathrm{~cm}$ primary mirror with a spatial resolution of about $0.2^{\prime \prime}$ at $550 \mathrm{~nm}$.

For our study, we used multi-wavelength observations from Hinode/SOT NFI (NarrowBand Filter Imager) and BFI (Broadband Filter Imager). This instrument measures the Stokes profiles of the FeI line at $630.2 \mathrm{~nm}$ with a spatial resolution $0.16^{\prime \prime}$. More precisely, the Lyot filter is set to a single wavelength in the blue wing of the line, typically $-120 \mathrm{~m} \AA$ for the $\mathrm{Fe}$ I magnetograms. Then images are taken at various phases of

1 The Hinode spacecraft, launched in 2006, was designed and is now operated by JAXA (Japanese space authorities) in cooperation with NASA (national aeronautics and space administration) and ESA (european space agency).

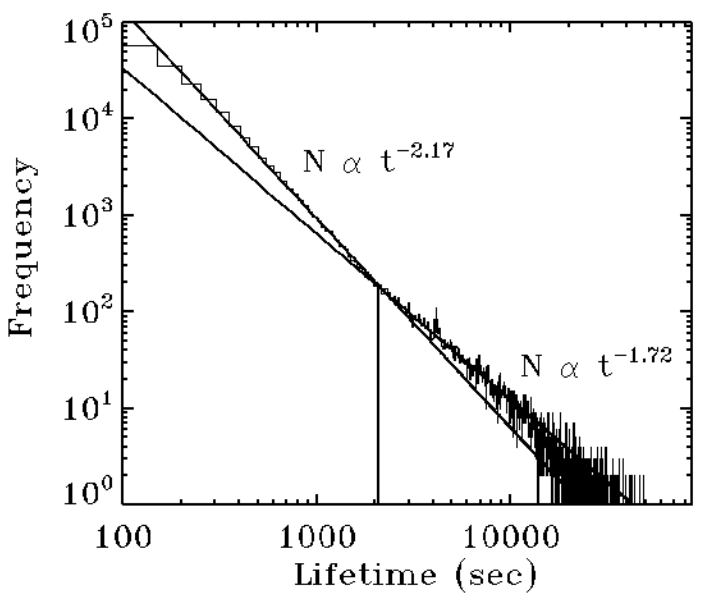

Fig. 1. Lifetime histogram of individual granules and TFGs for the full time sequence of $48 \mathrm{~h}$.

the rotating polarization modulator and added or subtracted into a smart memory area in the on-board computer. The units of both I and V images in both Level-0 and Level-1 are "data numbers" or DN. The BFI scans consist of time sequences obtained in the Blue Continuum (450.4 nm), G band (430.5 nm) and Ca II H (396.8 nm).

The observations were recorded continuously from 29 August 10:17 UT to 31 August 10:19 UT 2007, except for a $7 \mathrm{~min}$ interruption at the disk center on 30 August at 10:43 UT. The solar rotation is compensated for in order to observe exactly the same region of the Sun. The time step between two successive frames is $50.1 \mathrm{~s}$. The field of view with BFI observations is $111^{\prime \prime} .6 \times 111^{\prime \prime} .6$ with a pixel of 0.109 $(1024 \times 1024)$. After alignment, the useful field-of-view reduced to $100^{\prime \prime} \times 92^{\prime \prime}$.

To remove the effects of oscillations, we applied a subsonic Fourier filter. This filter is defined by a cone in the $k-\omega$ space, where $k$ and $\omega$ are spatial and temporal frequencies. All Fourier components such that $\omega / k \leq V=6 \mathrm{~km} \mathrm{~s}^{-1}$ are retained so as to keep only convective motion (Title et al. 1989). To detect TFGs, granules were labeled in time as described in Roudier et al. (2003).

\section{TFG detection and temporal properties}

3D analysis $(x, y, t)$ of the granular intensity field demonstrates that a significant fraction of the granules in the photosphere are organized in the form of TFGs (Roudier et al. 2003). A TFG consists of a family of repeatedly splitting granules, originating from a single granule. Since our data set is composed of two continuous sequences of $24 \mathrm{~h}$ separated by a gap of $7 \mathrm{~min}$, we first analyse separately each of the $24 \mathrm{~h}$ series and then connect them together to determine the evolution of TFGs over $48 \mathrm{~h}$.

As shown in Fig. 1, the distribution of the lifetime of the TFGs can be fitted by two power laws with exponents -2.17 and -1.72 for TFGs shorter and longer than 2100 s respectively. The second exponent, -1.72 , is identical to the one previously found with a $8.75 \mathrm{~h}$ image series obtained with the Swedish Vacuum Solar Telescope at La Palma (Roudier et al. 2003). Howewer, we find a different exponent for TFGs with lifetimes shorter than $2100 \pm 100 \mathrm{~s}$. This time scale seems to correspond to the longest isolated granules, which do not create a TFG. The Hinode data which are free of atmospheric seeing allows us to continuously follow the smallest granules. This explains why we detect more TFGs with shorter lifetimes than with the ground 


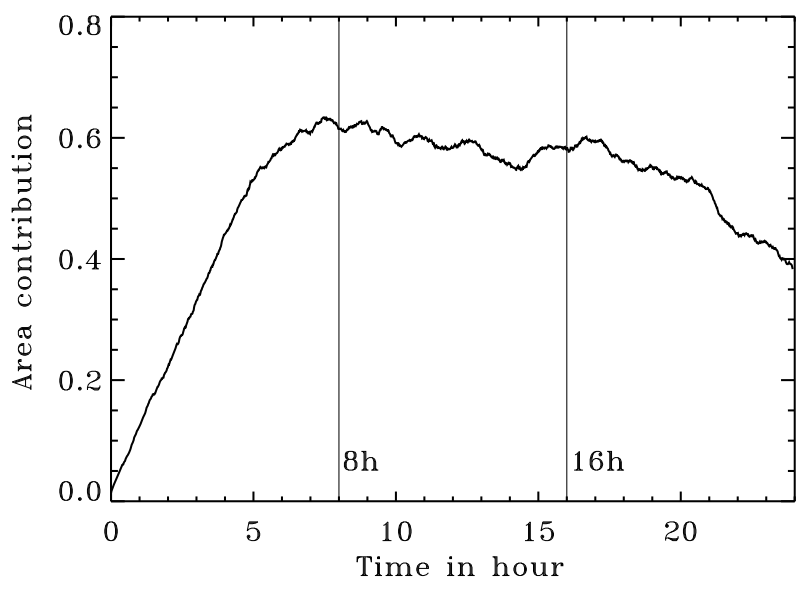

Fig. 2. Evolution of the percentage of granules belonging to TFGs existing longer than $8 \mathrm{~h}$.

based La Palma data. We conclude on the existence of two different kinds of TFGs characterized by their lifetime. The shorter lifetime TFGs appear scattered everywhere in the field of view between the longest TFGs. The slope of -1.72 for the longest TFGs is robust and easily reproducible; It would be interesting in the future to determine this slope using large cartesian numerical simulations of solar surface convection including scales up to the supergranulation scale (e.g. Georgobiani et al. 2007).

As some TFGs turn out to last longer than $24 \mathrm{~h}$, we connected the two time series, filling the $7 \mathrm{~min}$ gap with the following strategy: using the first time sequence, we compare the distribution of families at $t=24 \mathrm{~h}-7 \mathrm{~min}$ and at $t=24 \mathrm{~h}$. We observe that the area variation was not more than $3 \%$. We thus assumed that during the gap, the change was not larger and decided that families at $t=24 \mathrm{~h}$ could be reported as such at $t=24 \mathrm{~h}+7 \mathrm{~min}$, i.e. at the beginning of the second series. The alignment of the two sequences was also checked using the CaII and G-band data. In doing so, we managed to make an image sequence lasting $47^{\mathrm{h}} 23^{\mathrm{min}}$ and covering a field of view of $89^{\prime \prime} \times 103^{\prime \prime}$. Using this very long sequence, we could improve the statistics of data in Fig. 1, and found that the lifetime of TFGs could reach $43^{\mathrm{h}} 50^{\mathrm{min}}$ (within our field).

Although the number of TFGs decreases with lifetime, longlived TFGs cover a significant fraction of the solar surface. This is shown in Fig. 2, where the fraction of the total area covered by granules belonging to TFGs living more than $8 \mathrm{~h}$ is displayed using the first $24 \mathrm{~h}$-sequence. This plot shows that $60 \%$ of the area is covered by families living longer than $8 \mathrm{~h}$. This fraction steadily increases during the first $8 \mathrm{~h}$ because, during this period of time, many granules are still associated with families that were born before the beginning of the time-sequence and therefore appear to have a shorter lifetime. It also slightly decreases at the end of the sequence since the time window cuts the last born TFGs. If we now include more short-lived TFGs, reducing the lifetime threshold to $2 \mathrm{~h}$, then we find that the surface coverage increases up to $88 \%$. This is greater than the $62 \%$ that we found with the La Palma data. The difference is due to a better labeling of granules (no seeing and distortion), and a longer duration of the sequence allowing better statistics.

\section{Network and cork distributions}

\subsection{Velocity fields}

To further investigate the solar surface dynamics, we now focus our attention on the velocity fields and their transport properties. The velocity field is determined using the classical local correlation tracking (LCT) algorithm (e.g. Roudier et al. 1999) using a spatial window FWHM of $3^{\prime \prime}$ and a temporal window of $30 \mathrm{~min}$. The typical rms velocity is $300 \mathrm{~m} / \mathrm{s}$. As in Meunier et al. (2007) and Rieutord et al. (2008), we find that the probability distribution functions of divergence at scales larger than $10 \mathrm{Mm}$ show a net excess, compared to a Gaussian distribution, in the positive wing, suggesting intermittency in solar surface convection.

\subsection{Cork motions}

The transport properties at the surface of the turbulent velocity field are best illustrated using the evolution of an initially uniformly distributed passive scalar; the image is classically that of floating corks (Simon et al. 1994). The corks are deposited on the meshes of a regular grid at initial time $t=0$ and their surface distribution is followed during the whole sequence as they are advected by the horizontal velocity field. Figure 3 displays the corks distribution resulting from the evolution of a TFG depicted in Fig. 6. The isolated action of the velocity field within an isolated TFG leads to the advection of $90 \%$ corks at a mesogranular scale on a timescale of between two and four hours (flows are not taken into account outside of the isolated TFG). A comparison with the underlying families shows that they are simply expelled from a given family. The first snapshots of Fig. 4 clearly show this phenomenon. At later times, the corks remain more or less at the TFG boundaries as can be seen in Fig. 5. As we have shown in our previous paper (Roudier et al. 2003) (Figs. 11-13), TFGs are equivalent to a diverging flow at mesoscales. So, this scale is persistent up to $43 \mathrm{~h}$ corresponding, in the Hinode data, to the TFG itself or to some TFG branches for the longest living TFG. The large scale TFGs are probably the smallest supergranular sizes.

Investigating the mechanism of this fast transport, we found that exploding granules are the key features generating these motions. Figure 6 displays in detail the evolution of a family; the arrows indicate the location of the observed explosion during $1^{\mathrm{h}} 25^{\mathrm{min}}$. The evolution of the time derivative of the family area is plotted in Fig. 7. It demonstrates that each increase of the total area of this family is related to granule explosions whose number is quoted over each peak of the curve. Hence, we observe that the family grows in area by a succession of granule explosions, which are correlated in time, thereby expelling the corks.

Figure 4 also shows the connection of families and the supergranular scale. In the second frame of this figure we note the birth of a white family. Then, the successive frames show its evolution and its surface growing up to frame 9. In frame 10, one can see a new yellow TFG birth, which growth in the following frames and pushes the initial white TFG. We observe that the distribution of corks at the supergranular scale also results from the combined action of long-lived TFGs that are close spatially and more or less in the same phase of their evolution. Their mutual interactions tend to expulse corks of the area they cover on 3-4 h and concentrate the corks in the magnetic network, at supergranular scales in approximatively 6-8 h. One example of this evolution can be seen in our data (and movies) in the center of the field of view where two long-lived TFGs are competing for their development.

On longer timescales, long living families tend to structure the cork distribution either by successive birth at the same location, or by interaction of two or more competing TFGs which are located in close proximity and in the same phase of their evolution. Overall, the formation of this pattern depends on the TFG 


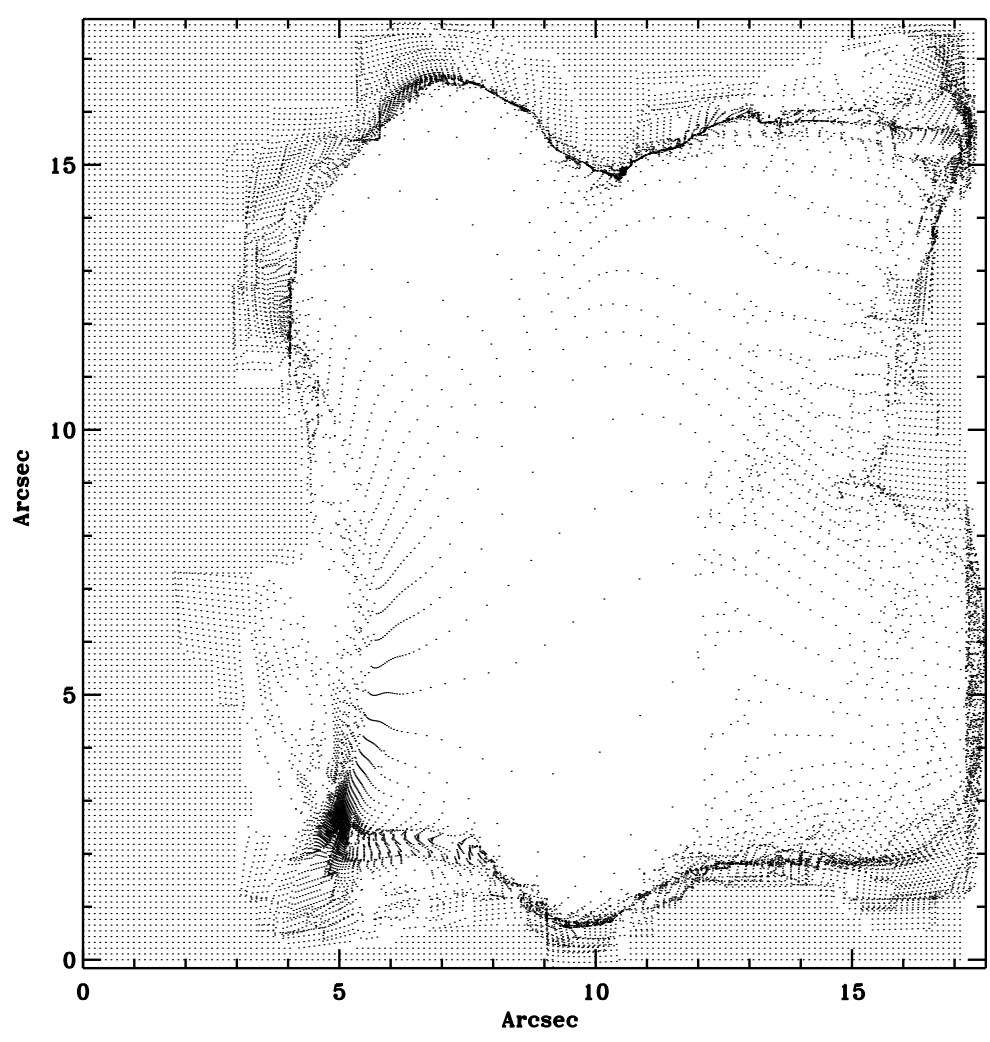

Fig. 3. Expulsion of corks of the TFG 2857 in approximatively $2 \mathrm{~h}$. The box has the size of the mesogranule scale.

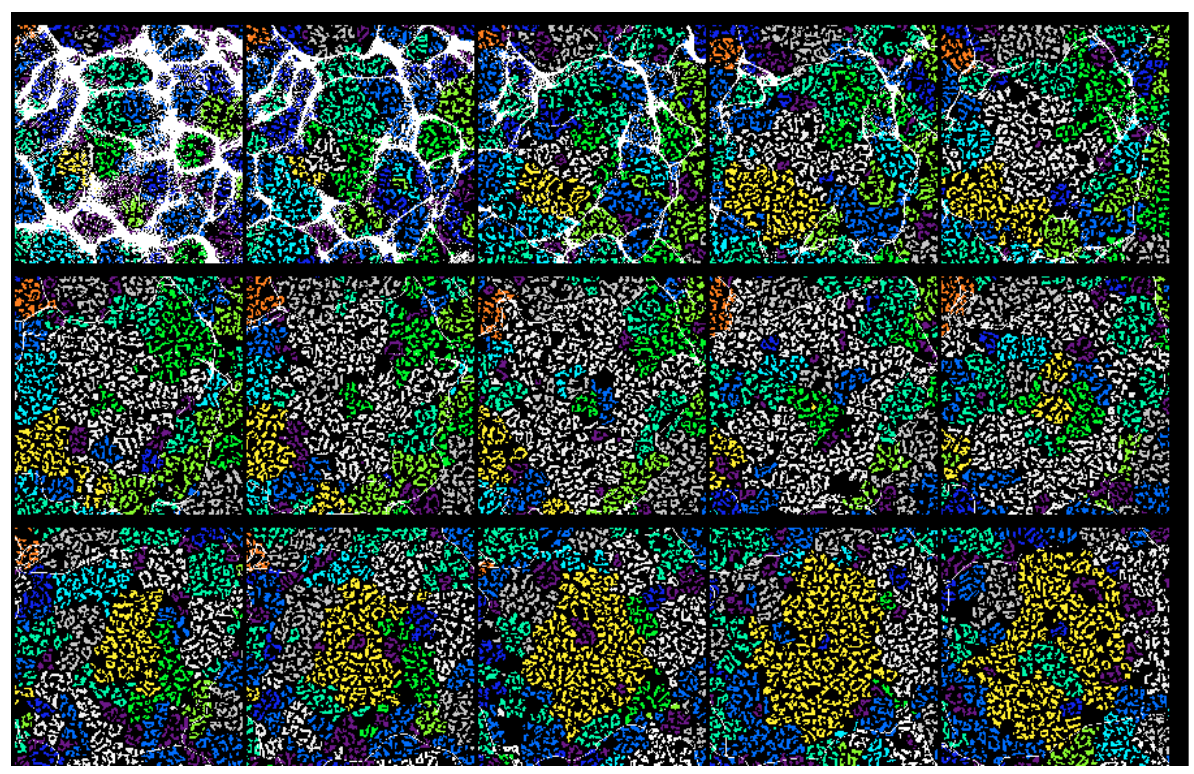

Fig. 4. Example of families evolution over $15 \mathrm{~h}$ with a time step of $1 \mathrm{~h}$. The field of view is $38.2^{\prime \prime} \times 39.9^{\prime \prime}$. White indicates a TFG lifetime of $24 \mathrm{~h}$, although yellow indicates a TFG lifetime of $20 \mathrm{~h}$. Green and blue stand for TFGs with a lifetime shorter than $18 \mathrm{~h}$. The families are colored (online) according to their lifetime: white ( $24 \mathrm{~h}$ and more), red ( 23 to $20 \mathrm{~h})$, orange (19 to $16 \mathrm{~h}$ ), yellow (15 to $12 \mathrm{~h}$ ), green (11 to $8 \mathrm{~h}$ ), blue ( 7 to $4 \mathrm{~h}$ ) and purple ( 3 to $1 \mathrm{~h}$ ). relative size and lifetime but seems to be mostly governed by long-lived TFGs.

\subsection{Corks and magnetic fields}

When longer time scales are considered it is well known that corks, like the magnetic field, concentrate on the supergranule boundaries (e.g. Rieutord et al. 2000; Krijger \& Roudier 2003). The quality of the data (free of atmospheric seeing) and the length of the time sequence allow us to provide a very clear illustration of this phenomenon. To determine the magnetic field distribution, we use Stokes $\mathrm{V}$ which is usually considered as a proxy for the radial magnetic field at the disk centers.
In Fig. 5, we see that magnetic fields, like corks, lie at the boundaries of granule families. In this figure, we clearly observe that the spatial evolution of the magnetic field (or corks) are related to the geometric evolution of the long lived TFG (shown here in orange). This demonstrates the physical action of the TFG on the magnetic field patch displacement over the solar surface and shows that plasma governs magnetic elements at the TFG scale in the quiet Sun. Figures 8 and 9 illustrate the same result at a larger scale when cork motion has been followed for $24 \mathrm{~h}$. Most of the corks $(70 \%)$ are still in the field-of-view at the end of each sequence. They exactly match the position of the highest magnetic flux concentration of the network, which lies on supergranule boundaries. The network boundaries defined by 

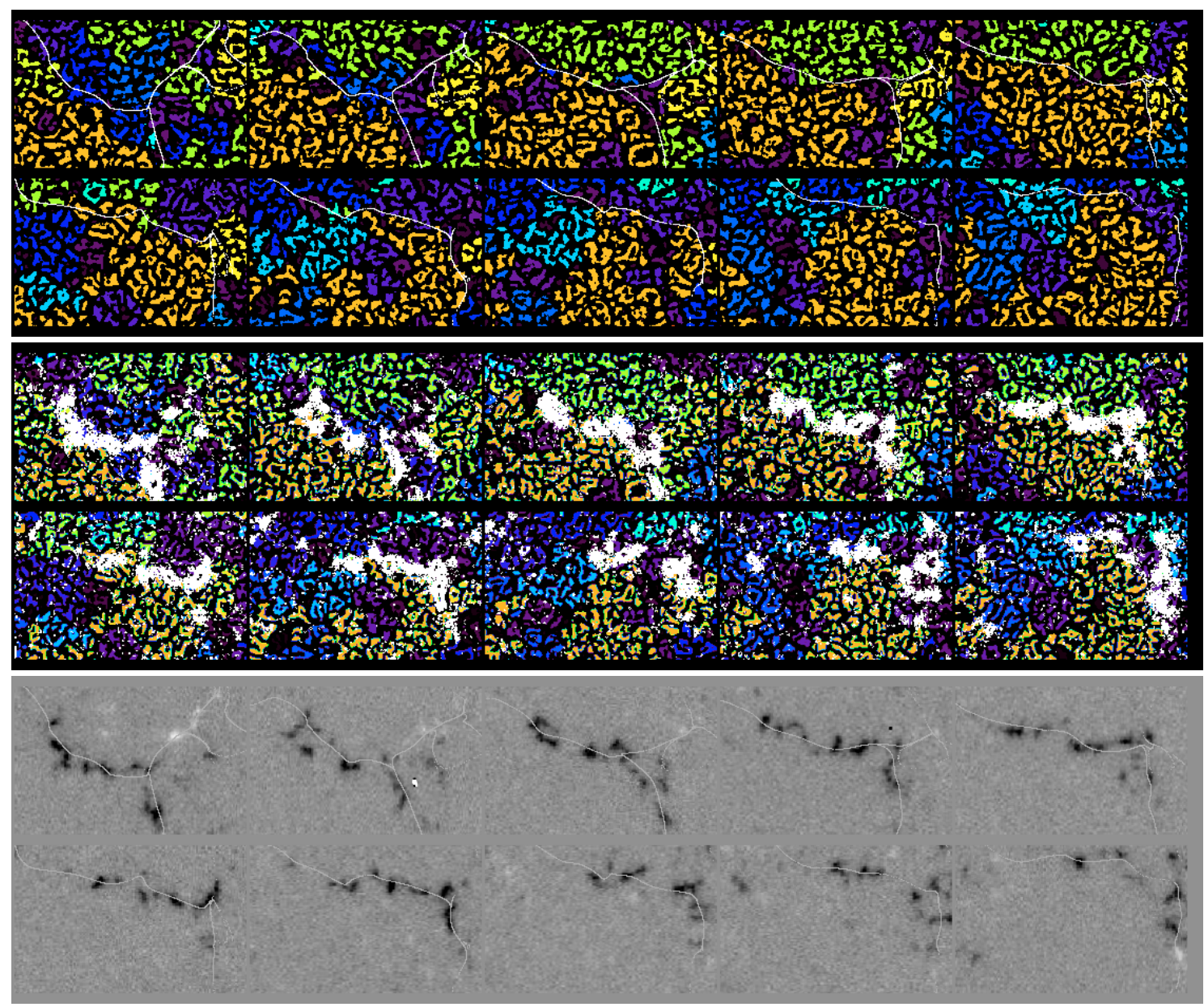

Fig. 5. Corks (top) and magnetic field modulus (middle) location with respect to families during $10 \mathrm{~h}, \delta t=1 \mathrm{~h}$. Corks and magnetic field (Stokes $V$ image) relative location (bottom). The field of view is $24^{\prime \prime} \times 15.3^{\prime \prime}$.

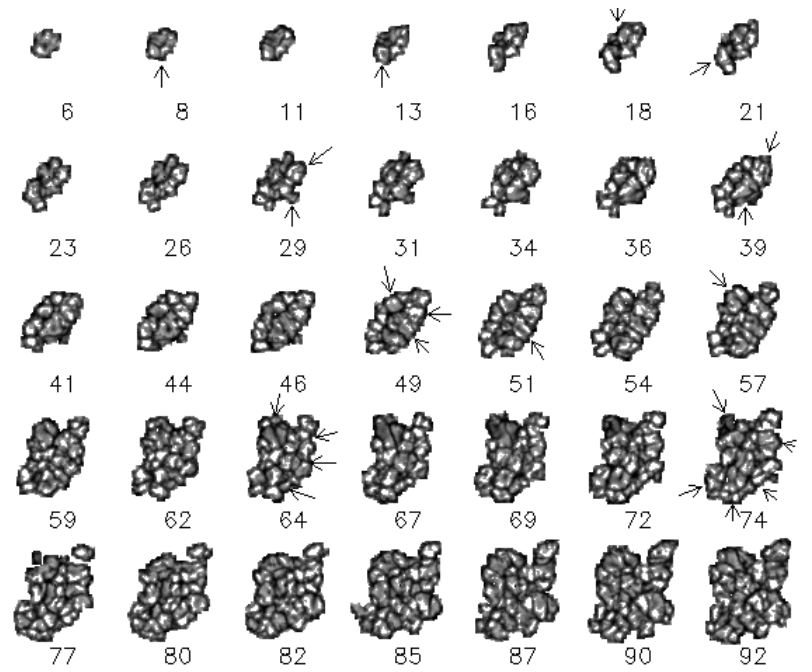

Fig. 6. Evolution of a TFG: time is given in minutes and arrows indicate the location of the granule explosion.

these patches are incomplete, with spatially intermittent sites of high magnetic flux density well correlated with sites of strong convergence.

If we further monitor the motion of the corks using the $48 \mathrm{~h}$ sequence, we find that after such a long time, $60 \%$ of the corks remain in the field-of-view, trapped in a small area of $\sim 10 \mathrm{Mm}^{2}$,

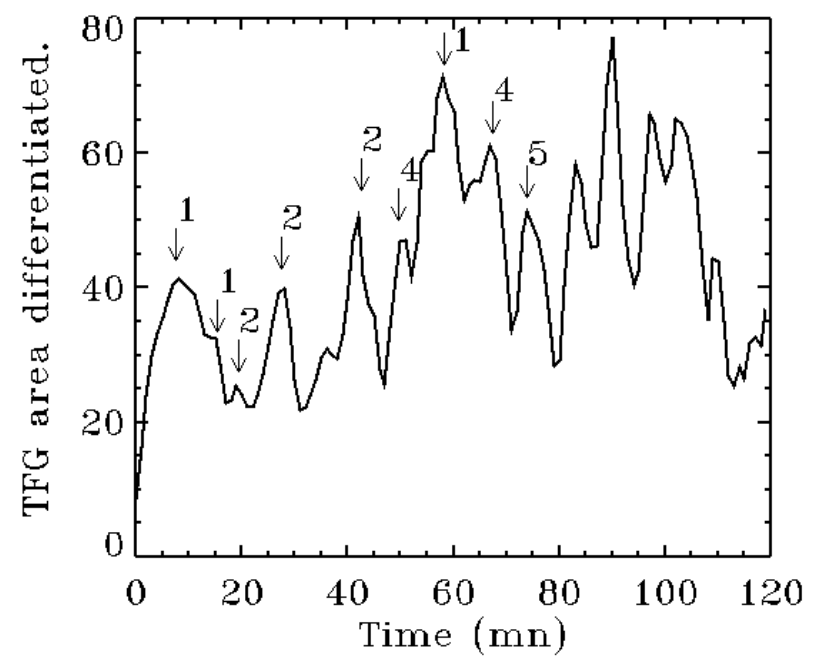

Fig. 7. Time evolution of the derivative of the area of the TFG shown in Fig. 6. Numbers refer to the number of explosions indicated by arrows in Fig. 6.

which also displays the most intense magnetic fields. This is illustrated in Figs. 10 and 11.

Furthermore, if we monitor the number of corks inside magnetic regions (we use a threshold of 45 SOT data number to detect the magnetic network, which corresponds, by using a raw 


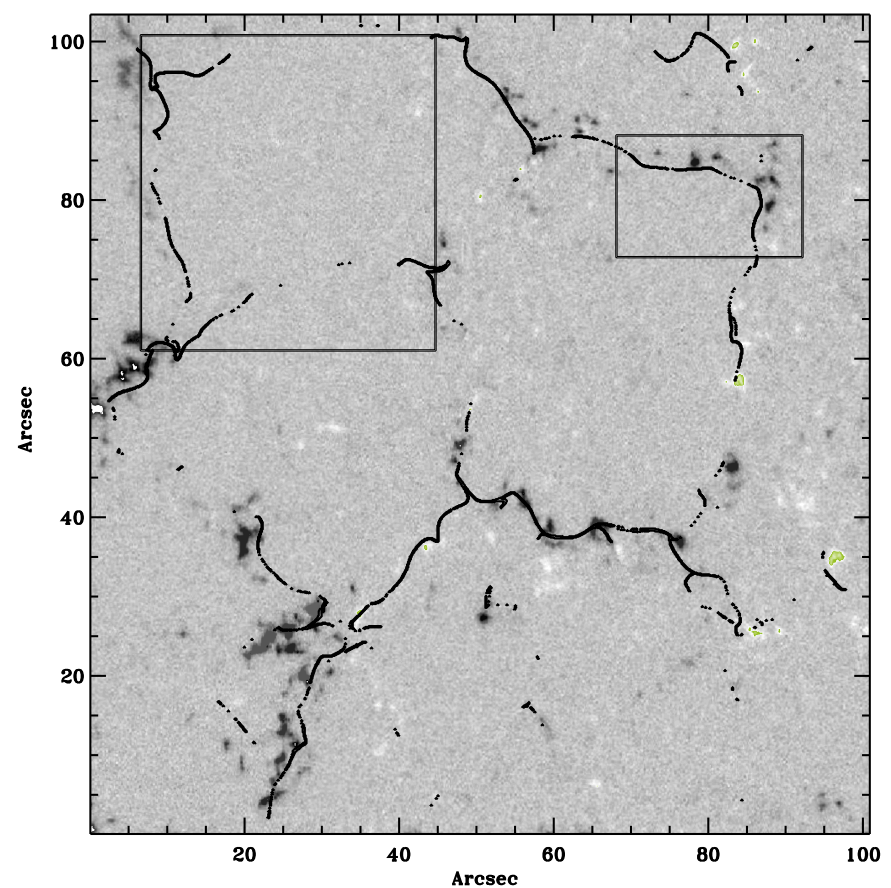

Fig. 8. Cork location at the end of the first time sequence of $24 \mathrm{~h}$ relatively to the longitudinal magnetic field (Stokes $V$ image). The left box shows the field of view of Fig. 4 and the right box indicates the field of view of Fig. 5.

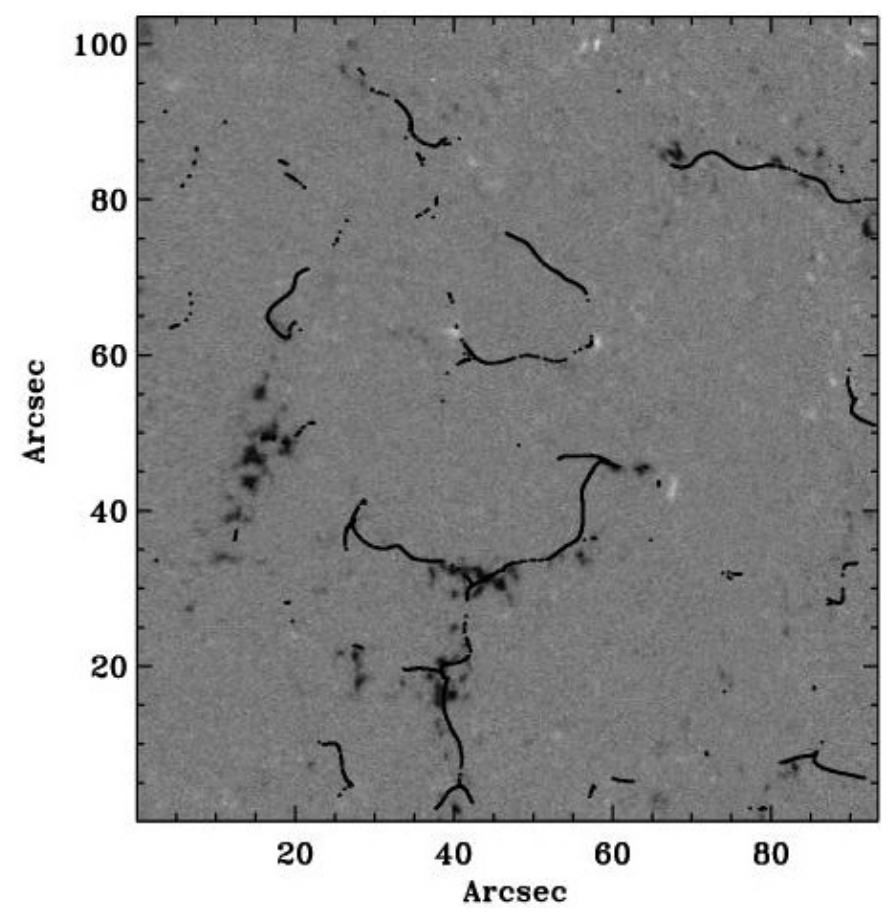

Fig. 9. Cork location at the end of the second time sequence of $24 \mathrm{~h}$ relative to the longitudinal magnetic field (Stokes $V$ image).

calibration described in Chae et al. 2007, to 248 Gauss), we find, as shown in Fig. 12, that $90 \%$ of the corks in the field end up in magnetic patches. This is particularly visible in movies ${ }^{2}$ of the evolution of both the magnetic field and the TFGs, which show that magnetic fields, squeezed between TFGs, follow their

\footnotetext{
${ }^{2}$ http://www.lesia.obspm.fr/ malherbe/papers/ Hinode2008/
}

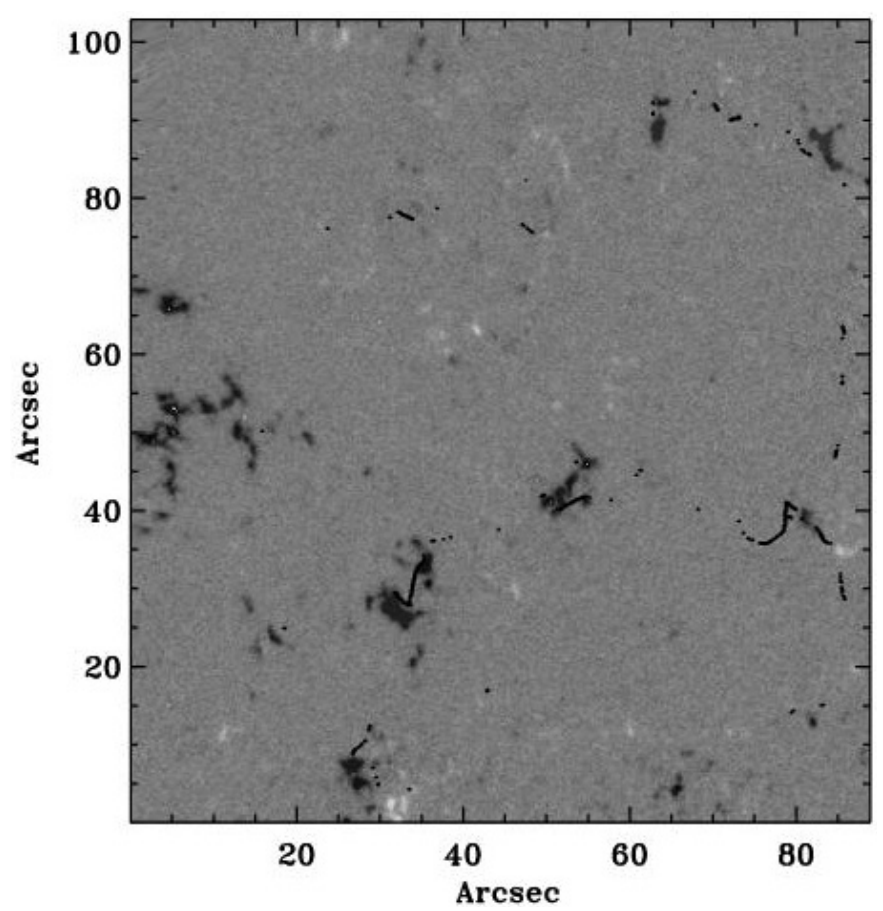

Fig. 10. Cork location at time $t=36 \mathrm{~h}$ relative to the longitudinal magnetic field (Stokes $V$ image).

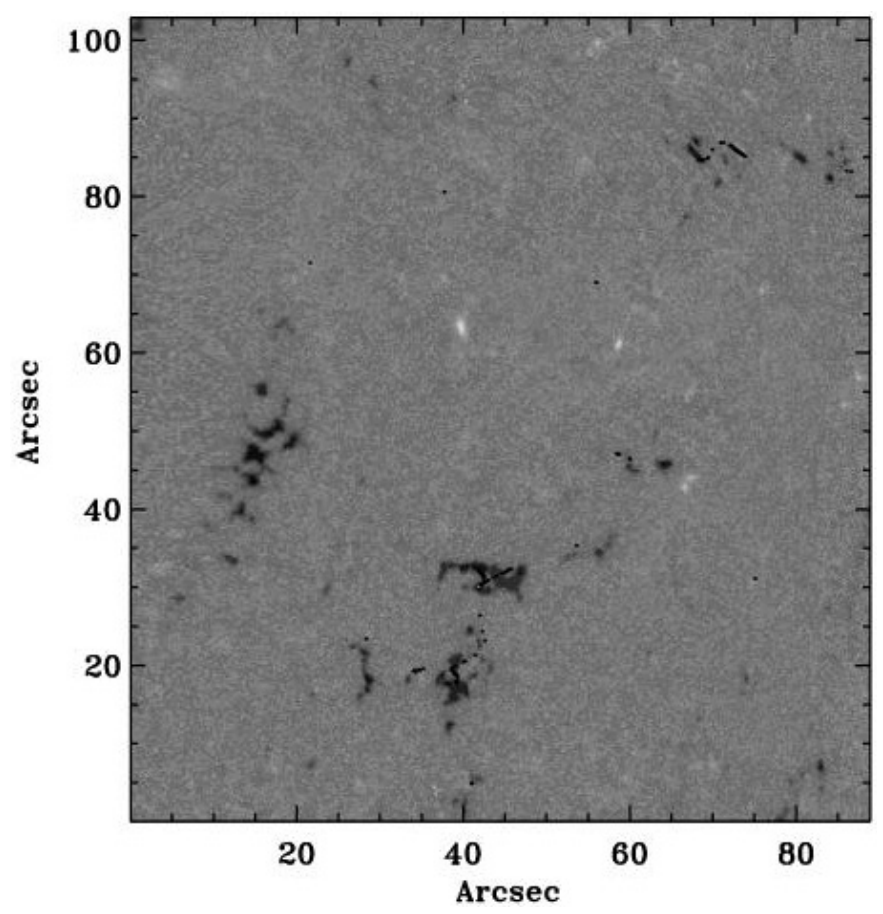

Fig. 11. Cork location at time $t=47 \mathrm{~h}$ relative to the longitudinal magnetic field (Stokes $V$ image).

displacement while drifting along their boundaries; at some time, they meet a vertex of TFG boundaries and become stabilized there, forming a patch.

As noted before, this patchy distribution of magnetic fields is closely associated with sites of strong flow convergence. These sites coincide with vertices of supergranular flow cells and presumably correspond to downflows, as required by mass conservation on the supergranular scale (Crouch et al. 2007). The observed stabilization of magnetic patches at supergranular scales 


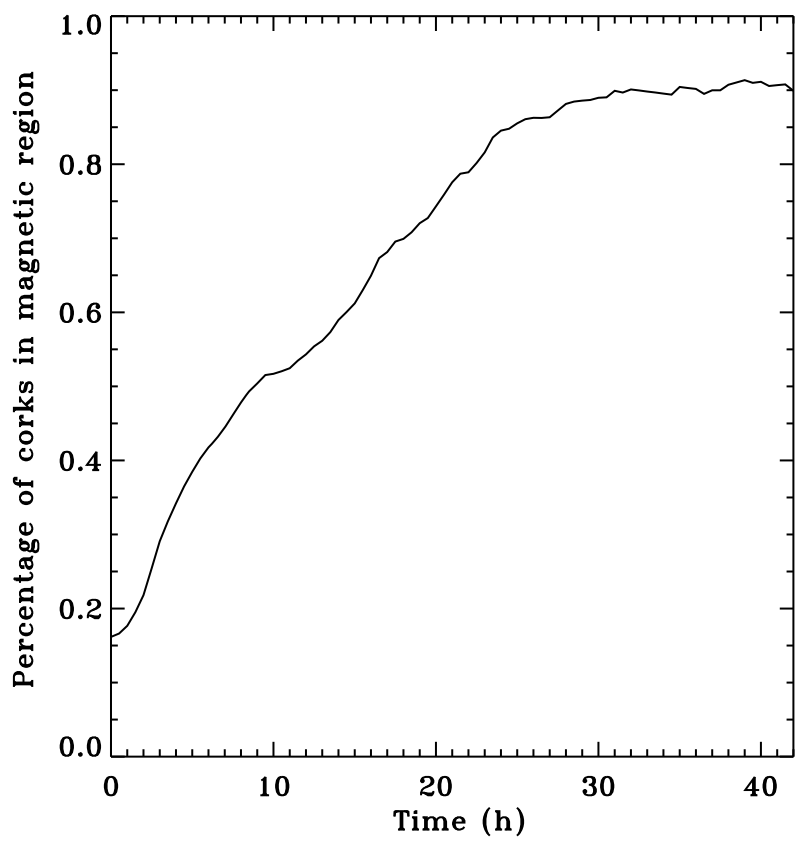

Fig. 12. Time evolution of the percent of corks located in the region where the longitudinal magnetic field magnitude is greater than 248 Gauss, which corresponds to of magnetic patches at the supergranule limits.

possibly indicates that the dynamical Lorentz force feedback provided by these concentrated patches is sufficiently strong to oppose the flow at scales comparable to the supergranular scale, which might explain the emergence of this particular scale in the velocity power spectrum of the quiet Sun.

\subsection{Horizontal turbulent diffusion}

Lastly, cork motion allows the measurement of the horizontal turbulent diffusivity at the surface of the Sun. The turbulent magnetic diffusion is an important quantity for mean-field dynamos (Brandenburg \& Subramanian 2005; Rieutord 2008) as it controls the timescale of the dynamo oscillations (in the case of a time-periodic dynamo). Thus several authors have evaluated this quantity. For instance, Simon et al. (1995), using a kinematic model tuned to match supergranulation properties, find diffusivities in the range 500 to $700 \mathrm{~km}^{2} \mathrm{~s}^{-1}$. With the very long time series that we have at our disposal, we can make a direct evaluation of this coefficient based on the precise plasma flow. However, because we use granules to determine the flow, the horizontal velocity fields are filtered at all scales below $2.5 \mathrm{Mm}$ (see Rieutord et al. 2001). Thus we give an estimate of the diffusivity imposed by the scales above $2.5 \mathrm{Mm}$.

As in other works, the diffusion coefficient is estimated from $D=\left\langle r^{2}\right\rangle / 4 t$, where $\left\langle r^{2}\right\rangle$ means the mean displacement of all particles (corks) at time $t$. As shown in Fig. 13 (solid line), after a rapid growth due to advection by the dominant mean flow (typically the mesogranular scale velocities), the diffusion coefficient seems to converge to a value of $430 \mathrm{~km}^{2} \mathrm{~s}^{-1}$. The diffusion coefficient also was computed with a starting reference position at time $6 \mathrm{~h}$ where the corks are at the mesoscale boarder. We observe that the cork motion along the mesoscale converge at around $29 \mathrm{~h}$ to the same value of the diffusion coefficient near a value of $430 \mathrm{~km}^{2} \mathrm{~s}^{-1}$. This indicates that corks and patches of corks are always in movement due to the TFG evolution. The figure shows, for the first time, the importance of using long time

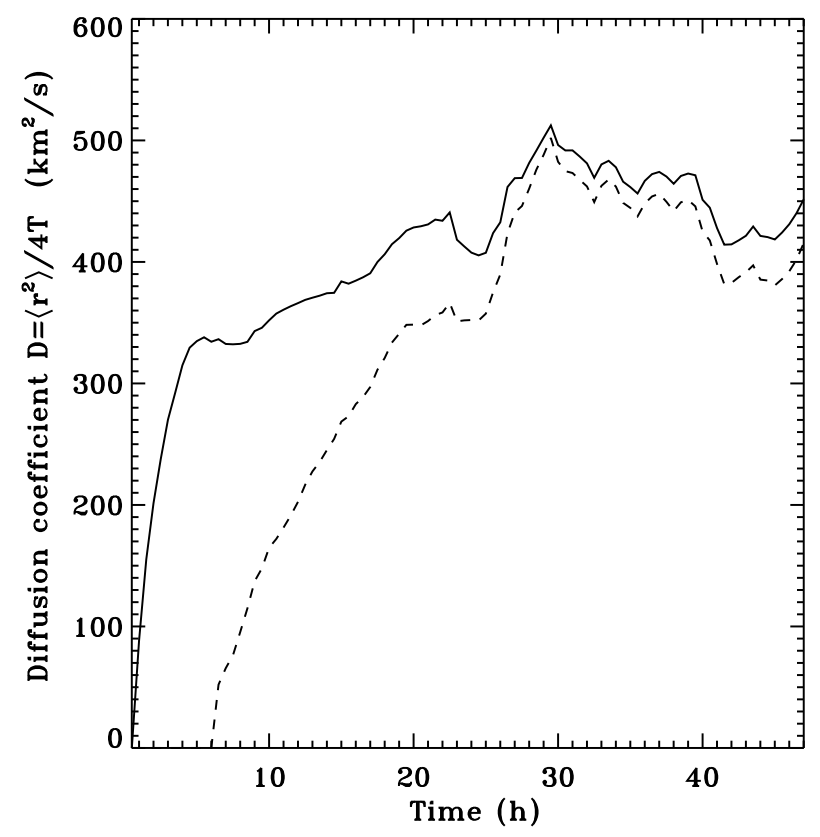

Fig. 13. Diffusion coefficient over $48 \mathrm{~h}$ (solid line) and the diffusion coefficient computed from $6 \mathrm{~h}$ to $48 \mathrm{~h}$ with a starting reference position at time $6 \mathrm{~h}$, when the corks are at the mesoscale border (dashed line).

series for this estimate. Saturation is indeed obtained only after $24 \mathrm{~h}$. With shorter time sequences we could expect a growth beyond the present value. Finally, even though this may be a coincidence, we note that the implied diffusion timescale over the solar magnetic belts, namely $\left(\lambda R_{\odot}\right)^{2} / 430$, is 11 yrs $(\lambda \simeq 32)$.

\section{Discussion and conclusion}

Thanks to the Solar Optical Telescope onboard Hinode, we have been able to monitor with high spatial and temporal resolution, free of atmospheric seeing, a region of the Sun's photosphere for two days. The field was wide enough to contain 4 supergranules and thus to examine their internal dynamics.

Using the TFG concept we could quantify the temporal and spatial correlations in the evolution of granules. The typical size of mature TFGs suggest that they trace some mesoscale rising plumes. In the previous debate on the specificity of mesogranulation as a genuine scale of sub-photospheric convection rather then a mere extension of granular flow (Straus \& Bonaccini 1997; Rieutord et al. 2000), TFGs point towards the genuine scale. In this scenario, granulation would be the boundary layer of these flows. However, since solar convection is driven by the strong cooling of the Sun's surface, it may still be that mesoscale flows are forced by the granulation layer. At this stage, it is clear that numerical simulations are necessary to better understand the connection between the dynamics of the surface layers and that of the deeper ones.

From our analysis, we observe that most of the granules visible on the solar surface belong to the long-lived TFGs, indicating that these families structure the flow. The evolution of floating corks demonstrates that the final state of passively advected quantities like weak magnetic elements is a patchy distribution on the boundaries of a supergranule, which correspond presumably to stable downward flows. The stabilization of magnetic patches in these areas suggests that supergranulation could be an emergent length scale building up as weak small-scale magnetic elements are advected and concentrated 
by TFG flows, occasionally colliding and aggregating to form larger and stronger magnetic patches, eventually resisting the flow dynamically. The temporal evolution of magnetic elements, corks and TFGs are available at http://www. lesia.obspm. $\mathrm{fr} /$ malherbe/papers/Hinode2008/.

Two kind of interactions between TFGs are mainly observed to diffuse the corks to the limit of supergranular scales. The first one corresponds to successive long-lived TFG births at the same location, whose combined motions sweep corks to network scales; parts of older TFGs, with a classical mesoscale, are pushed away by new TFGs, thereby contributing to the formation of a larger scale comparable to the supergranular scale. The second process is the common action of two or more close long-lived TFGs born more or less at the same time, which push corks to the network limit. The temporal co-spatiality between magnetic fields and corks in our time sequence analysis implies that the evolution of the distribution of magnetic fields is clearly driven by TFGs on the Sun's surface. A relation between mesoscale flows and the distribution of magnetic elements was also reported by Domínguez Cerdeña (2003), Domínguez Cerdeña et al. (2003), Rouppe van der Voort et al. (2005) and Lites et al. (2007).

Finally, the length of the monitoring time window (48 h) allowed us to directly estimate the diffusion coefficient induced by horizontal motion at scales larger than $2.5 \mathrm{Mm}$. We found a value of $430 \mathrm{~km}^{2} \mathrm{~s}^{-1}$, which might be helpful to design meanfield dynamo models.

Satellites like Hinode/SOT or the future SDO as well as large field-of-view ground based instruments like CALAS at Pic du Midi now enable us to study the full complexity of the interactions of multiple scales of convection and magnetic fields at the solar surface. In this paper we have shown that identifying and following coherent structures like TFGs can help us understand the detailed physics of these interactions and how they relate to the solar cycle.

Acknowledgements. We are grateful to the Hinode team for the possibility to use their data. Hinode is a Japanese mission developed and launched by ISAS/JAXA, collaborating with NAOJ as a domestic partner, NASA and STFC (UK) as international partners. Scientific operation of the Hinode mission is conducted by the Hinode science team organized at ISAS/JAXA. This team mainly consists of scientists from institutes in the partner countries. Support for the post-launch operation is provided by JAXA and NAOJ (Japan), STFC (UK), NASA, ESA, and NSC (Norway). This work was (partly) carried out at the NAOJ Hinode Science Center, which is supported by the Grant-in-Aid for Creative Scientific Research "The Basic Study of Space Weather Prediction" from MEXT, Japan
(Head Investigator: K. Shibata), generous donations from Sun Microsystems, and NAOJ internal funding. We thank the CNRS/INSU for support of the oneyear sabbatical of D. Brito at LATT (while member of LGIT, Université JosephFourier, CNRS in Grenoble). This work was supported by the Centre National de la Recherche Scientifique (C.N.R.S., UMR 5572), by the Programme National Soleil Terre (P.N.S.T.).

\section{References}

Aiouaz, T. 2008, ApJ, 674, 1144

Brandenburg, A., \& Subramanian, K. 2005, Phys. Rep., 417, 1

Chae, J., Moon, Y.-J., Park, Y.-D., et al. 2007, PASJ, 59, 619

Crouch, A. D., Charbonneau, P., \& Thibault, K. 2007, ApJ, 662, 715

DeRosa, M. L., \& Toomre, J. 2004, ApJ, 616, 1242

Domínguez Cerdeña, I. 2003, A\&A, 412, L65

Domínguez Cerdeña, I., Sánchez Almeida, J., \& Kneer, F. 2003, A\&A, 407, 741

Georgobiani, D., Zhao, J., Kosovichev, A. G., et al. 2007, ApJ, 657, 1157

Giacalone, J., Jokipii, J. R., \& Mazur, J. E. 2000, ApJ, 532, L75

Gizon, L., Duvall, T. L., \& Schou, J. 2003, Nature, 421, 43

Hassler, D. M., Dammasch, I. E., Lemaire, P., et al. 1999, Science, 283, 810

Ichimoto, K., Tsuneta, S., Suematsu, Y., et al. 2004, in Society of Photo-Optical Instrumentation Engineers (SPIE) Conf., Optical, Infrared, and Millimeter Space Telescopes, ed. J. C. Mather, 5487, 1142

Krijger, J. M., \& Roudier, T. 2003, A\&A, 403, 715

Lisle, J. P., Rast, M. P., \& Toomre, J. 2004, ApJ, 608, 1167

Lites, B., Socas-Navarro, H., Kubo, M., et al. 2007, PASJ, 59, 571

Meunier, N., Tkaczuk, R., Roudier, T., \& Rieutord, M. 2007, A\&A, 461, 1141

Ossendrijver, M. 2003, A\&ARv, 11, 287

Rast, M. P. 2003, ApJ, 597, 1200

Rast, M. P., Lisle, J. P., \& Toomre, J. 2004, ApJ, 608, 1156

Rieutord, M., Roudier, T., Malherbe, J. M., \& Rincon, F. 2000, A\&A, 357, 1063

Rieutord, M. 2008, C. R. Physique, 9, 757

Rieutord, M., Roudier, T., Ludwig, H.-G., Nordlund, Å., \& Stein, R. 2001, A\&A, 377, L14

Rieutord, M., Meunier, N., Roudier, T., et al. 2008, A\&A, 479, L17

Rincon, F., \& Rieutord, M. 2003, in SF2A-2003: Semaine de l'Astrophysique Francaise, ed. F. Combes, D. Barret, T. Contini, \& L. Pagani, 103

Rincon, F., Lignières, F., \& Rieutord, M. 2005, A\&A, 430, L57

Roudier, T., Rieutord, M., Malherbe, J., \& Vigneau, J. 1999, A\&A, 349, 301

Roudier, T., Lignières, F., Rieutord, M., Brandt, P. N., \& Malherbe, J. M. 2003, A\&A, 409, 299

Rouppe van der Voort, L. H. M., Hansteen, V. H., Carlsson, M., et al. 2005, A\&A, 435, 327

Schou, J. 2003, ApJ, 596, L259

Simon, G. W., Brandt, P. N., November, L., Scharmer, G. B., \& Shine, R. A. 1994, in Solar Surface Magnetism, ed. R. Rutten \& C. Schrijver (Kluwer), 261

Simon, G. W., Title, A. M., \& Weiss, N. O. 1995, ApJ, 442, 886

Straus, T., \& Bonaccini, D. 1997, A\&A, 324, 704

Suematsu, Y., Tsuneta, S., Ichimoto, K., et al. 2008, Sol. Phys., 249, 197

Title, A. M., Tarbell, T. D., Topka, K. P., et al. 1989, ApJ, 336, 475 